\title{
Non-canonical Notch signaling represents an ancestral mechanism to regulate neural differentiation
}

\author{
Michael J Layden ${ }^{1}$ and Mark Q Martindale $2^{2^{*}}$
}

\begin{abstract}
Background: Cellular differentiation is a critical process during development of multicellular animals that must be tightly controlled in order to avoid precocious differentiation or failed generation of differentiated cell types. Research in flies, vertebrates, and nematodes has led to the identification of a conserved role for Notch signaling as a mechanism to regulate cellular differentiation regardless of tissue/cell type. Notch signaling can occur through a canonical pathway that results in the activation of hes gene expression by a complex consisting of the Notch intracellular domain, SuH, and the Mastermind co-activator. Alternatively, Notch signaling can occur via a non-canonical mechanism that does not require $\mathrm{SuH}$ or activation of hes gene expression. Regardless of which mechanism is being used, high Notch activity generally inhibits further differentiation, while low Notch activity promotes differentiation. Flies, vertebrates, and nematodes are all bilaterians, and it is therefore unclear if Notch regulation of differentiation is a bilaterian innovation, or if it represents a more ancient mechanism in animals.

Results: To reconstruct the ancestral function of Notch signaling we investigate Notch function in a non-bilaterian animal, the sea anemone Nematostella vectensis (Cnidaria). Morpholino or pharmacological knockdown of Nvnotch causes increased expression of the neural differentiation gene NvashA. Conversely, overactivation of Notch activity resulting from overexpression of the Nvnotch intracellular domain or by overexpression of the Notch ligand Nvdelta suppresses NvashA. We also knocked down or overactivated components of the canonical Notch signaling pathway. We disrupted NvsuH with morpholino or by overexpressing a dominant negative NvsuH construct. We saw no change in expression levels for Nvhes genes or NvashA. Overexpression of Nvhes genes did not alter NvashA expression levels. Lastly, we tested additional markers associated with neuronal differentiation and observed that non-canonical Notch signaling broadly suppresses neural differentiation in Nematostella.
\end{abstract}

Conclusions: We conclude that one ancestral role for Notch in metazoans was to regulate neural differentiation. Remarkably, we found no evidence for a functional canonical Notch pathway during Nematostella embryogenesis, suggesting that the non-canonical hes-independent Notch signaling mechanism may represent an ancestral Notch signaling pathway.

Keywords: Notch, Nvnotch, Nematostella vectensis, Cellular differentiation, Evolution

\footnotetext{
* Correspondence: mamartin@whitney.ufl.edu

${ }^{2}$ Whitney Laboratory for Marine Bioscience, University of Florida, St

Augustine, FL 32080, USA

Full list of author information is available at the end of the article
}

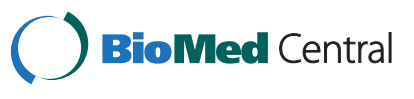

(c) 2014 Layden and Martindale; licensee BioMed Central Ltd. This is an Open Access article distributed under the terms of the Creative Commons Attribution License (http://creativecommons.org/licenses/by/4.0), which permits unrestricted use, distribution, and reproduction in any medium, provided the original work is properly credited. The Creative Commons Public Domain Dedication waiver (http://creativecommons.org/publicdomain/zero/1.0/) applies to the data made available in this article, unless otherwise stated. 


\section{Background}

Metazoan development requires a mechanism to control the balance between pools of cells that are able to differentiate into distinct specialized cell types and cells that remain undifferentiated to contribute to growth or differentiate at a later time. Identifying the mechanisms that regulate this balance provides insights into the evolution of animal developmental programs and clues as to the putative molecular changes that underscored the emergence of metazoans from single-celled ancestors. Functional studies have identified the Notch signaling pathway (described below) as a conserved regulator of cellular differentiation, but this is only known from bilaterian animals. There are at least four metazoan lineages that diverged prior to the emergence of bilaterians. They are the ctenophores, poriferans, placozoans, and cnidarians, with cnidarians being the most closely related to bilaterians [1-4].

Notch signaling is implicated as a regulator of cellular differentiation in multiple bilaterian tissue types including neural, blood, epidermal, endothelial, muscle, and bone [5-10]. A well known and studied example of Notch regulation of differentiation is in bilaterian neurogenesis. During the formation of the Drosophila ventral nerve cord, cells with high Notch activity suppress the formation of a neuroblast progenitor cell in favor of maintaining undifferentiated neural ectoderm fate $[11,12]$. Similarly, in vertebrate neurogenesis, high Notch activity in neural stem cells acts to maintain a neural stem cell fate identity, while low notch activity in daughter cells promotes neuronal differentiation $[8,13]$. In both vertebrate and invertebrate neurogenesis, Notch inhibits neurogenesis by repressing the expression of proneural gene transcription factors [11,13-15]. Proneural gene transcription factors are basic helix-loop-helix transcription factors that belong to either the achaete-scute or atonal gene families [15].

There are two mechanisms by which Notch can regulate differentiation. They are the canonical [16] and noncanonical pathway $[16,17]$. The core minimal components shared by both pathways are the notch receptor, delta ligand, and the $\gamma$-secretase and ADAM protease cleavage complexes $[9,16]$. Additional core components required specifically for canonical Notch signaling are hes, suH, smrt, and mastermind $[9,18,19]$. Both canonical and non-canonical pathways are typically initiated by the binding of Delta to the Notch receptor, which induces cleavage and release of the Notch intracellular domain by Adam protease and $\gamma$-secretase cleavage events. In the canonical pathway, the Notch intracellular domain interacts with $\mathrm{SuH}$ and displaces the SMRT co-repressor normally bound to $\mathrm{SuH}$ and recruits the Mastermind transcriptional co-activator. This complex then induces expression of the hes genes, which regulate expression of Notch targets, such as the proneural genes $[9,16,18]$. Noncanonical Notch signaling bypasses interactions with $\mathrm{SuH}$ and activation of hes gene expression, to regulate target gene expression via alternative mechanisms [16,20].

Genomic analyses of core conserved Notch components suggest that the core Notch pathway evolved specifically in the metazoan lineage. notch, delta, and hes homologs do not exist outside of the metazoans [19,21], and all five major animal clades possess a Notch homolog. The ctenophores are the only non-bilaterians lacking a definitive Delta ligand, although they possess many Delta-like proteins that could potentially activate Notch ligands [1,19], and, recently, Delta-like genes have been identified to activate Notch in bilaterians [22]. Of the remaining core conserved genes, the members of the $\gamma$-secretase complex and ADAM proteases all predate the metazoan divergence [19]. Key regulatory components of the canonical Notch pathway were not present until the emergence of the cnidarian-bilaterian common ancestor. The $s u H$ gene evolved prior to the earliest metazoans, but the mastermind co-activator evolved in the cnidarian-bilaterian ancestor [19], and the SMRT corepressor is not present outside of the bilaterian lineage $[1,3,4,19,23,24]$. Thus, although Notch signaling evolved early in the metazoan lineage, it is unclear if the canonical or non-canonical pathway represents the ancestral Notch signaling mechanism.

One way to determine the evolution of a particular signaling pathway is to determine how it functions in phylogenetically informative extant animals that allow reconstruction of the ancestral role(s) of the pathway at deep evolutionary nodes within the animal phylogeny. However, gene-specific functional studies addressing Notch signaling in non-bilaterian metazoans is currently lacking. Characterization of the expression patterns of Notch signaling components and pharmacological disruption of $\gamma$-secretase implicate Notch as a regulator of differentiation in the non-bilaterians [25-28]. First, in the poriferan Amphimedon queenslandica, Amqdelta homologs are expressed in differentiating cell types throughout development [25]. In the cnidarians, treatment with DAPT, which inhibits $\gamma$-secretase cleavage of the Notch intracellular domain $[26,28,29]$, increases expression of differentiated cell markers (particularly neuronal markers) [26]. One of these markers is NvashA, which is an achaete-scute gene family homolog known to regulate embryonic neurogenesis in Nematostella [30]. Marlow and coworkers [26] also investigated the role of $\mathrm{NvsuH}$ on development of the cnidocytes, which are the stinging cells in Nematostella, using a splice blocking morpholino (MO) against the $N v s u H$ gene and a dominant negative construct. They found that mature cnidocytes were lacking in Nematostella planula when $\mathrm{NvsuH}$ function was reduced and that this phenotype was similar to the reduction in cnidocytes resulting from treating animals with DAPT [26]. However, in this study the authors did not compare other phenotypes resulting from 
DAPT treatment with a disruption of $N v s u H$. DAPT treatment has been found to inhibit maturation, but not specification, of cnidocytes in polyps of the hydrozoan cnidarian Hydra [28]. Taken together, the previous studies in nonbilaterians suggest that Notch signaling played a role in regulating the process of neuronal cell differentiation in the cnidarian-bilaterian ancestor, but the lack of detailed gene-specific studies does not clarify if the canonical or non-canonical Notch signaling pathway represents the ancestral mechanism of Notch signaling.

Here we take advantage of the ability to conduct functional genetic experiments in the cnidarian sea anemone Nematostella vectensis to characterize the role of Notch signaling during embryonic development. We show that Notch activity in Nematostella suppresses expression of NvashA-dependent neural differentiation markers [30], and that the suppression of NvashA-dependent neural markers occurs via specific inhibition of NvashA expression by Nvnotch. We also show that Notch activity broadly inhibits expression of neuronal differentiation markers for other neural cell types in the Nematostella embryo. Although some components of canonical Notch signaling are present in the Nematostella genome, our experiments indicate that inhibition of differentiated cell markers occurs via the non-canonical (Nvhes-independent) mechanism during embryonic development.

\section{Methods}

\section{Genes used in this study}

The genes used in this study were previously published $[26,30,31]$.

\section{Embryo manipulations and in situ analysis}

All embryos were grown to either early gastrula stages, by raising animals for 24 hours post-fertilization (hpf) at $17^{\circ} \mathrm{C}$, or to late gastrula stages, by raising animals for 24 hpf at $25^{\circ} \mathrm{C}$. All fixation, in situ probe synthesis, and in situ hybridizations were carried out as previously described [30,32,33]. Images were obtained on a Zeiss Imager M2 in conjunction with the Axiocam HRc and ZenPro software (Carl Zeiss LLC, Thornwood, NY, USA). For gastrula stage analysis, $10 \mu \mathrm{M}$ DAPT treatment was begun $3 \mathrm{hpf}$ as previously described [26]. For larval stage analysis of DAPT-treated animals, animals were allowed to grow to desired stage (either 24 or $48 \mathrm{hpf}$ at $25^{\circ} \mathrm{C}$ ) and then, animals were incubated in $10 \mu \mathrm{M}$ DAPT for 24 hours.

\section{mRNA injections}

The Nvnicd fusion construct was generated by PCR amplifying the intracellular domain of Nvnotch using (Forward 5' CACCATGGTTGTTGTGCTCGCAGGCG GTAAG 3' and Reverse 5' GTCTGATAATAACTCCA CTATGTC 3') PCR primers. The PCR product was then cloned Nvnicd in frame and $5^{\prime}$ to the venus coding sequence using the Gateway cloning vector system (Invitrogen, Carlsbad, CA, USA). Full length Nvdelta was amplified using the (Forward 5'CACCATGCAGC TACTACCACTCCAGCCATCAC 3' and Reverse 5' ATATTTCCACTTCCACTTCTTGCCAG 3') primers and cloned in frame $5^{\prime}$ to the venus coding sequence. Nvhes 2 and Nvhes 3 constructs were cloned using (Forward 5' CACCATGGAAAAAATGCGGAGGGCGAG 3' and Reverse 5' TCAAATTGTCCTCCCCATTCAC $3^{\prime}$ ) and (Forward 5' CACCGCCGTTGACTGCATCG ATAGC 3' and Reverse 5' TCACCATGGGCGCCAC AGTG) PCR primers, respectively. They were both cloned in frame to the $3^{\prime}$ end of the venus coding sequence. Injection concentration of the Nvnicd:venus was $300 \mathrm{ng} / \mu \mathrm{l}$. Injection concentration of Nvdelta:venus was $900 \mathrm{ng} / \mu \mathrm{l}$. Injection concentrations of venus:hes 2 and venus:hes 3 were $300 \mathrm{ng} / \mu \mathrm{l}$ and $150 \mathrm{ng} / \mu \mathrm{l}$, respectively. We injected the previously published NvashA: venus and NvsuHDN:venus mRNA as described previously (Layden and colleagues [30] and Marlow and colleagues [26]). mRNA was prepared and injected as previous described [30,34]. Animals were sorted prior to analysis to identify embryos expressing the Venus reporter protein and to eliminate the non-expressing animals.

\section{Morpholino injections}

Fluorescein labeled NvashA translation-blocking $\mathrm{MO}$ was injected as published [30]. $\mathrm{NvSuH}$ splice-blocking $\mathrm{MO}$ was injected, and splice blocking was observed as previously described [26]. An Nvnotch splice-blocking MO (5' GTCCTTTGATTTCGTACCTCATGGA 3') (GeneTools Inc., Philomath, OR, USA) that results in a truncation of the Nvnotch intracellular domain and Nvdelta splice-blocking MO (5' GCGACCTGACAAGAACAGTGAAGTC 3') (GeneTools Inc.) that removes the exon containing the MNNL domain were designed and injected at $1 \mathrm{mM}$ and $600 \mathrm{nM}$, respectively. Splice-blocking efficiency was estimated using PCR and DNA electrophoresis to observe shifts in the size of the wild-type or morphant mRNA. A control MO (5' AGAGGAAGAATAACATACCCTGTCC $\left.3^{\prime}\right)$ was also injected at a concentration of $1 \mathrm{mM}$ and gene expression was compared to uninjected control animals. Animals were sorted after injection to eliminate the uninjected animals as indicated by the lack of fluorescence.

\section{Quantification of cell number}

To count the number of NvashA-expressing cells we mounted animals with the aboral end up, visualized using the $10 \times$ objective on the Zeiss Imager M2 (Carl Zeiss LLC). We normalized the focal plane by focusing on the most superficial level of the aboral ectoderm and then counted the total number of visible cells. 


\section{Quantitative PCR analysis}

RNA isolation and quantitative (q)PCR analyses were conducted as previously described [30]. Nvactin, Nvef1B, and Nvatpsynthase house-keeping genes were used to normalize fold change in qPCR experiments. All qPCR primers used have been previously described [26,30,35]. Each qPCR analysis was repeated in triplicate pools of embryos injected in independent sessions. Based on previous studies, we consider a fold change greater than 1.5 meaningful. We often fail to detect changes in expression via alternative approaches for fold-changes less than 1.5.

\section{Results}

\section{Nvnotch and Nvdelta spatiotemporal expression is consistent with that of a regulator of cellular differentiation}

Previous studies showed that Nvnotch and Nvdelta are expressed in tissues known to be undergoing differentiation in late gastrula through juvenile polyp developmental stages [26]. However, multiple studies suggest differentiation in Nematostella is first observed in the early gastrula when expression of neural genes NvashA [30] and Nvelav $[31,36]$ are detected. We tested for both Nvnotch and Nvdelta expression by mRNA in situ hybridization in early gastrula animals (Figure 1). Initially, Nvnotch and Nvdelta expression is distributed in a "salt and pepper" pattern (Figure $1 \mathrm{~A}, \mathrm{C}$ ), meaning that the cells that are expressing

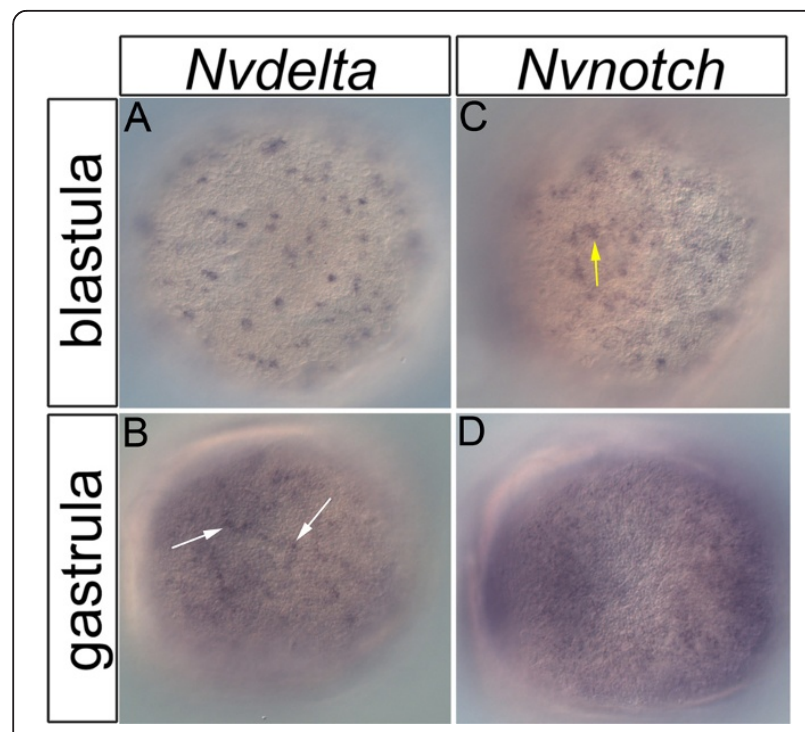

Figure 1 Nvnotch and Nvdelta embryonic expression. Expression of Nvdelta $(\mathbf{A}, \mathbf{B})$ and Nvnotch $(\mathbf{C}, \mathbf{D})$ is shown at early gastrula $(\mathbf{A}, \mathbf{C})$ and late gastrula (B,D) stages. Nvdelta is expressed in a "salt and pepper" expression pattern at early gastrula (A), and ubiquitously expressed at late gastrula (B), though there are cells enriched for Nvdelta in the late gastrula (B, arrows). Clusters of cells distributed in a "salt and pepper" pattern express Nvnotch in the early gastrula stages $(\mathbf{C})$. By late gastrula, Nvnotch appears to have low-level ubiquitous expression (D). Images are lateral views taken from a superficial focal plane; oral is to the left.
Nvdelta and Nvnotch are distributed throughout the ectoderm and appear like pepper granules mixed into a pile of salt. The Nvnotch "salt and pepper" pattern is slightly variable in that it appears patchy as if there are clusters of Nvnotch expressing cells distributed in the "salt and pepper" pattern (Figure 1C, yellow arrow). The expression of both genes expands over time, and both genes are ubiquitously expressed by the late gastrula stage (Figure 1B,D). Interestingly, within the ubiquitous Nvdelta expression, there is a "salt and pepper" distribution of cells that appear enriched for Nvdelta (Figure 2B, white arrows). Based on the spatiotemporal expression patterns previously reported [26] and extended here, Nvnotch and Nvdelta expression is consistent with the earliest onset of cellular differentiation.

\section{Nvnotch inhibits expression of the neurogenic transcription factor NvashA}

To determine if Notch signaling in Nematostella functions to regulate cellular differentiation, we chose to characterize the effects of NvNotch activity on the expression of the previously identified neural differentiation gene NvashA.

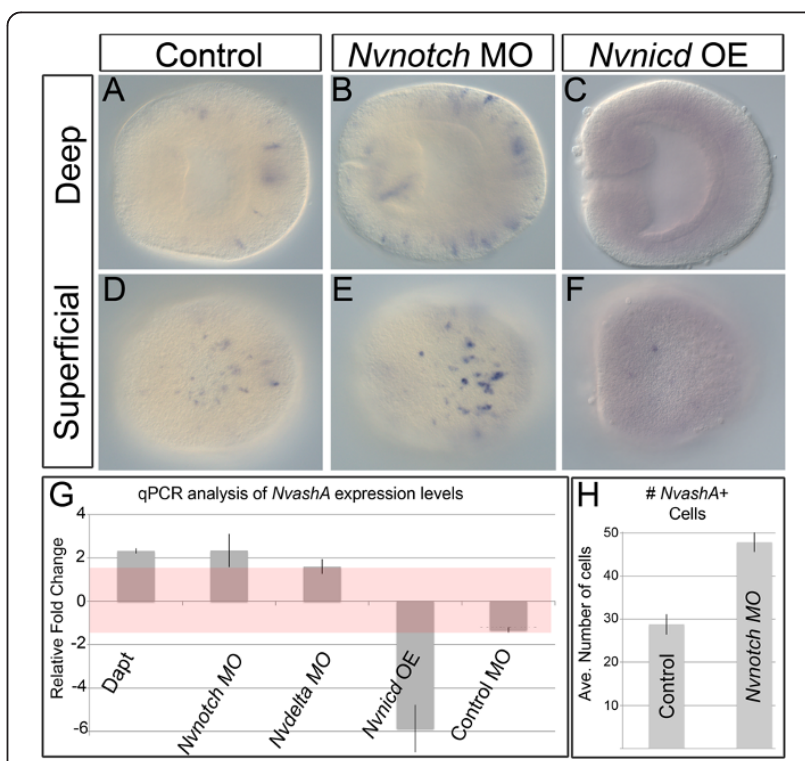

Figure 2 Activation of Nvnotch suppresses NvashA expression. Images of animals stained for NvashA by in situ hybridization are shown (A-F). All images are lateral views with oral to the left. The relative focal plane is indicated to the left of each row of images. Animals with control wild-type Notch activity $(\mathbf{A}, \mathbf{D})$, with Notch activity reduced by injection of a Nvnotch morpholino $(\mathrm{MO}) \mathbf{( B , E )}$, and with Notch activity overactivated by overexpression (OE) of the Nvnotch intracellular domain (Nvnicd) (C,F) are shown. (G) Quantitative (q)PCR analysis of the relative expression of $N$ vashA is compared in animals with reduced Notch activity (DAPT, Nvnotch MO, Nvdelta MO) and increased Notch activity (Nvnicd OE), and to animals injected with a control MO. The red rectangle indicates a relative fold change of -1.5 to 1.5 , which we consider to correspond with no change in expression level. $\mathbf{( H )}$ Quantification of the average number of NvashA-positive cells counted in the aboral domain (see Methods). $N \geq 20$ animals counted for each treatment. 
Previous reports showed that continuous treatment with DAPT for 72 hours resulted in an upregulation of NvashA [26], but this study did not characterize earlier DAPT phenotypes. We assayed gastrula treated with $10 \mu \mathrm{M}$ DAPT for NvashA expression by mRNA in situ hybridization and qPCR (Additional file 1; Figure 2). NvashA expression levels increased by approximately two-fold in DAPT treated animals (Additional file 1; Figure 2G). Because DAPT does not directly inhibit Notch signaling, we were concerned that the DAPT NvashA phenotype may be caused by a disruption of a pathway other than Notch. To confirm that Notch signaling specifically inhibits NvashA expression, we generated splice-blocking MOs directed against the Nvdelta ligand and the Nvnotch receptor (Additional file 2A). The splice-blocking Nvnotch MO results in Nvnotch mRNAs containing stop codons that result in a premature truncation of the Notch intracellular domain (data not shown). Injection of the Nvnotch splice-blocking MO resulted in a cell that appeared to express relatively higher levels of NvashA compared to control (compare Figure 2A and D to $\mathrm{B}$ and $\mathrm{E}$ ), a two-fold increase in NvashA expression measured by qPCR (Figure 2G), and a $60 \%$ increase in the number of $N v a s h A$ positive cells (Figure $2 \mathrm{H}$ ). The similar increase in NvashA expression observed in DAPTtreated and Nvnotch MO-injected animals suggest that the NvashA phenotype observed in DAPT-treated animals is specifically due to inhibition of NvNotch. A splice-blocking MO generated against NvDelta generates a miss-spliced transcript that encodes an Nvdelta transcript only missing the MNNL domain present in the extracellular region of the protein (Additional file 2A; data not shown). Injection of the Nvdelta splice-blocking MO results in an approximate 1.6-fold increase in NvashA expression (Figure $2 \mathrm{G}$ ). This demonstrates that NvNotch and NvDelta are both required to repress NvashA in the embryonic ectoderm.
To further confirm that Notch activity functions to repress $N v a s h A$, we used two approaches to overactivate Notch activity. First, we mimicked constitutively active Notch by injecting an mRNA encoding the Nvnotch intracellular domain fused in frame to the venus coding sequence (Nvnicd:venus) [37]. We observed NvNicd:Venus nuclear localization (Additional file 2D), and a nearly complete repression of NvashA expression as detected by mRNA in situ hybridization (Compare Figure 2A and D to $\mathrm{C}$ and $\mathrm{F}$ ), and an approximate six-fold reduction in NvashA levels as detected by qPCR (Figure 2G). Second, we overactivated Notch activity by injecting mRNA encoding for the full length Nvdelta gene fused to the venus reporter (Nvdelta:venus). Overexpression of Nvdelta showed lower levels of NvashA expression by mRNA in situ hybridization (Figure 3). We observed weak NvashA expression in $57 \%$ of the Nvdelta:venus injected animals (Figure $3 \mathrm{~A}, \mathrm{~B}$ ) and an approximate three-fold reduction in Nvash $A$ expression as measured by qPCR (Figure $3 C$, light grey bar). To determine if the suppression of NvashA by Nvdelta required NvNotch, we treated Nvdelta:venus injected animals with DAPT. Treating Nvdelta:venus injected animals with DAPT resulted in a two-fold upregulation of NvashA (Figure $3 \mathrm{C}$, dark grey bar). This is consistent with the previously observed phenotypes following DAPT treatment and NvNotch MO injection (Figure 2), and suggests that NvNotch acts to inhibit NvashA expression when activated by interactions with NvDelta.

\section{Notch activity suppresses neurogenesis through repression of NvashA expression}

To determine if changes in NvashA levels downstream of Notch activity correspond to changes in NvashA-dependent neurogenesis, we assayed for changes in expression of the previously identified NvashA neural target genes

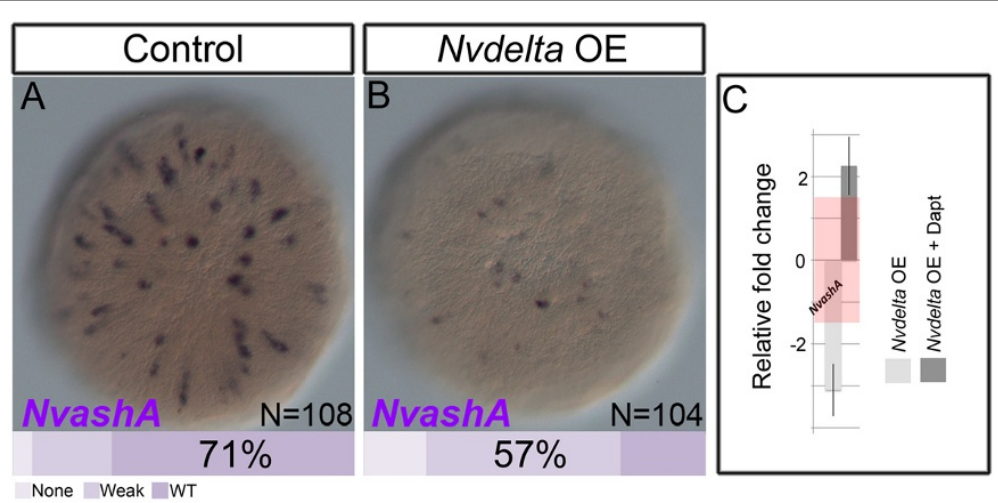

Figure 3 Nvdelta activates Nvnotch activity to suppress NvashA. (A-B) Shown are Aboral views of NvashA expression in control (A) and Nvdelta overexpressing (OE) (B) animals. Phenotypic classes were scored as no expression, weak, wild-type (WT) levels, and strong expression. The key is shown in the image and bars at the base of each image represent the percentage of animals in each phenotypic class. (C) Relative fold change of $\mathrm{NvashA}$ and previously identified Nvasha neural gene targets and in animals overexpressing Nvdelta (light grey bars) and animals that are overexpressing Nvdelta and treated with DAPT (dark grey bars). Red rectangle denotes relative fold change -1.5 to 1.5 , which corresponds to no change in relative expression. 
[30]. Overactivation of Notch activity by either injection of the full length Nvdelta:venus or injection of the Nvnicd:venus construct resulted in a dramatic downregulation of NvashA neural target genes (Figure 4A,D,G, dark blue bars; Additional file 3, light grey bars). Coinjection of NvashA:venus mRNA with the Nvnicd:venus mRNA was sufficient to suppress the reduction of neural gene expression phenotype resulting from overactivation of Notch activity (Figure 4C,F,G, light blue bars). Many of the NvashA:venus Nvnicd:venus co-injected embryos assayed by in situ hybridization showed neural gene expression phenotypes consistent with the increased number of neurons observed when NvashA is expressed alone (Figure 4C,F) [30]. Treatment with DAPT increased the levels of neural gene expression (Figure 4A, dark orange bars). Co-injection of the NvashA translationblocking MO [30] suppresses the DAPT induced upregulation of neural gene expression (Figure 4A, light orange bars). These data suggest that Notch activity suppresses NvashA-dependent neurogenesis primarily
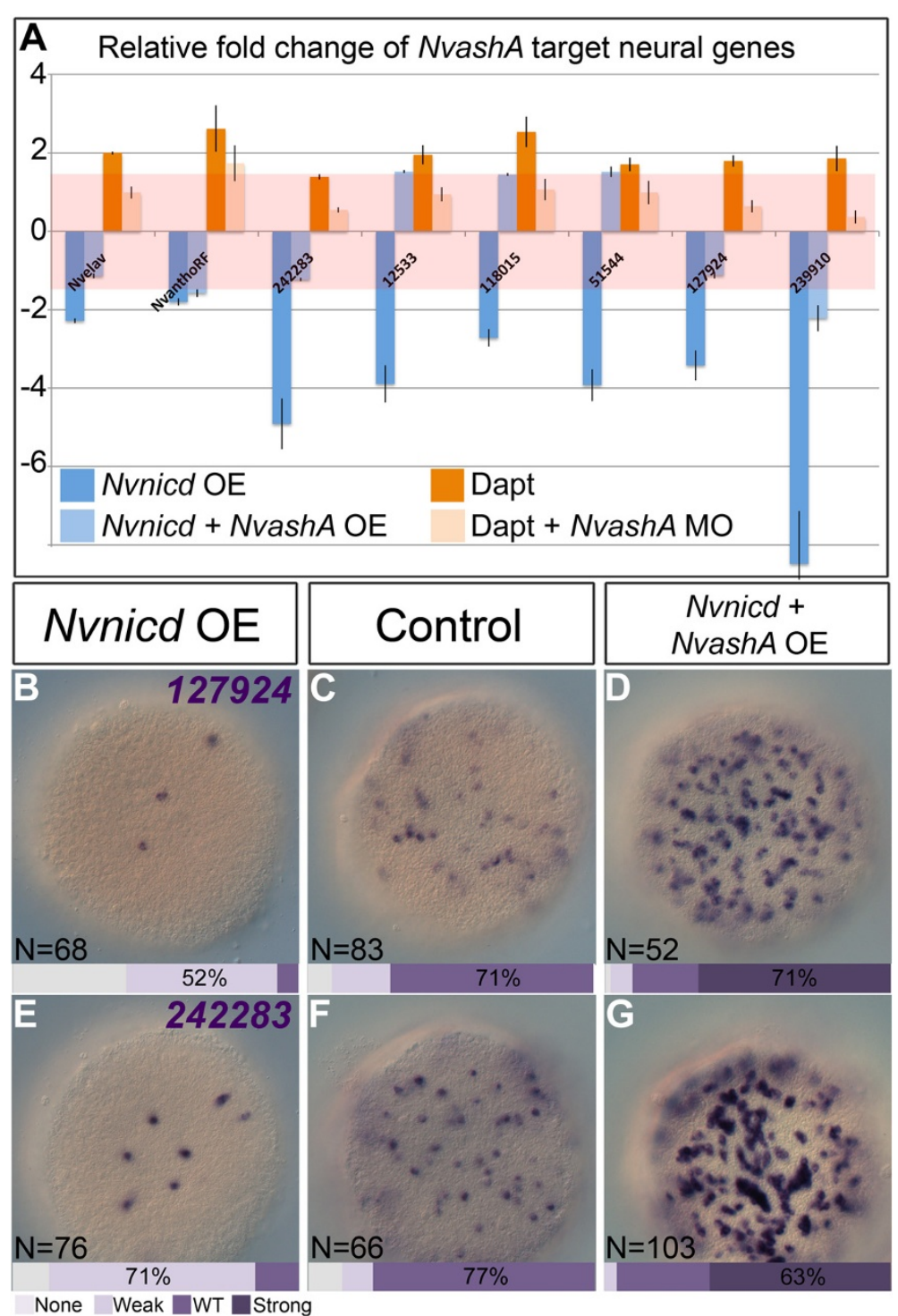

Figure 4 Nvnotch suppresses neurogenesis by regulating NvashA expression. (A) Relative expression levels of NvashA target genes in animals with overexpressing (OE) Nvnicd (dark blue bars), overexpressing Nvnicd and NvashA (light blue bars), animals treated with DAPT (dark orange bars), and DAPT treated animals injected with the NvashA morpholino (MO) (light orange bars). Red rectangle represents relative fold change -1.5 to 1.5 , which corresponds to no change in relative expression. Each treatment was repeated at least three times. (B-G) Aboral views of mRNA in situ images from two NvashA neural target genes are shown. Animals with overactive Nvnotch $\mathbf{( B , E )}$, control $(\mathbf{C}, \mathbf{F})$, and both overactive Nvnotch and overactive NvashA (D,G) are shown. Animals in (B-G) were quantified into phenotypic classes based on having no, weak, wild-type (WT)-like, or strong expression levels. The key is shown in the image and bars at the base of each image represent the percentage of animals in each phenotypic class. 
through the specific inhibition of NvashA expression rather than broadly targeting downstream genes expressed in differentiating neurons.

\section{Post-embryonic treatment with DAPT increases NvashA expression in the larval ectoderm and endoderm}

We wanted to test whether Notch activity regulates NvashA at later developmental stages independently of the earlier roles described above. In order to disrupt Notch signaling at later stages without disrupting Notch signaling at early stages we opted to use DAPT treatments. Although DAPT treatment may not specifically disrupt Notch signaling, the increase in NvashA following treatment with DAPT or injection of the Nvnotch MO in the embryo are identical (Figure 1), which suggests the DAPT NvashA phenotype is due to a disruption of Notch signaling. We performed two DAPT treatments (Figure 5). The first treatment began at the late gastrula stage and continued for 24 hours into the early planula larval stages (Figure 5A-C). We detected NvashA expression in the forming pharynx (Figure 5A, arrow), in a "salt and pepper" pattern in the ectoderm (Figure 5A, inset), and some weak staining in a "salt and pepper" pattern within the endoderm in control planulae (Figure 5A, arrow head). Treatment with DAPT resulted in an increase in pharyngeal staining (Figure 5B, arrow) and an increase in the number of ectodermal cells expressing NvashA (Figure 5B, inset). It was difficult to be certain that endodermal NvashA was increased because of the strong ectodermal expression, but it appears as if there is an expansion of NvashA expression in the endoderm as well. We were also able to classify animals into groups of animals having no, weak, normal wild-type, or strong NvashA expression for both control and DAPTtreated animals. In control animals, approximately $70 \%$ of the animals had wild-type levels of NvashA expression, and only approximately $10 \%$ of the animals had strong expression of NvashA. In DAPT-treated animals $90 \%$ of the animals displayed the strong expression phenotype. We also observed a three-fold increase in NvashA expression in DAPT-treated animals by qPCR (Figure $5 \mathrm{C}$ ). We also treated animals with DAPT from 48 to $72 \mathrm{hpf}$, which ensured animals were all within the larval stages of development during the treatment (Figure 5D-F). NvashA expression in control $72 \mathrm{hpf}$ planulae was detected in the pharynx and forming mesentery structures (Figure 5D, arrow) and in a "salt and pepper" endodermal pattern. We

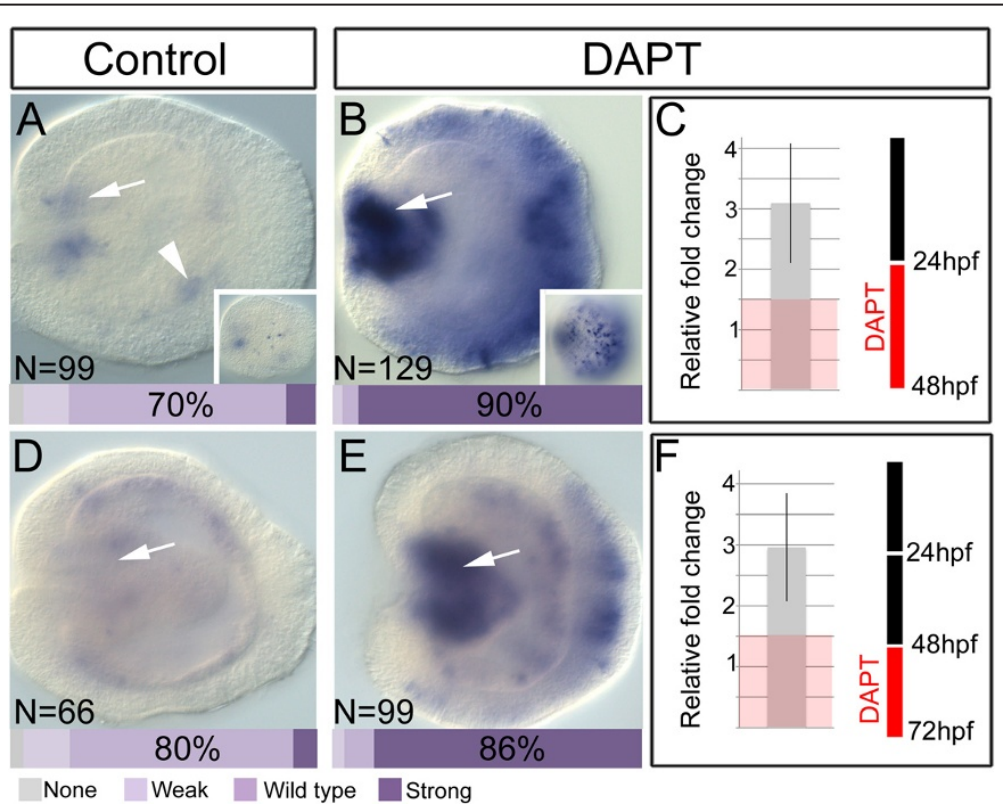

Figure 5 DAPT treatment increases NvashA expression in the planula larva. (A-C) Forty-eight hours post fertilization (hpf) animals either treated with control DMSO (A) or with DAPT (B-C). (A) NvashA expression in control animals is detected in the developing pharynx (arrow), in the endoderm (arrowhead), and in the ectoderm (inset). (B) Treatment with DAPT increases NvashA expression in each tissue. (C) Quantitative (q)PCR analysis reveals a three-fold increase in the relative levels of NvashA in DAPT-treated animals. (D-F) Seventy-two hpf animals either treated with control DMSO (D) or with DAPT (E-F). (D) NvashA expression in control animals is detected in the developing pharynx (arrow) and in the endoderm. (E) Treatment with DAPT increases NvashA expression in each tissue. (F) qPCR analysis reveals a three-fold increase in the relative levels of NvashA in DAPT-treated animals. The key in (C) and (F) shows that animals were grown in normal 1/3X sea water (black line between time intervals) or in the presence of DAPT (red line between time intervals). Animals in (A,B,D,E) were quantified into phenotypic classes based on having no, weak, wild-type-like, or strong expression levels. The key is shown in the image and bars at the base of each image represent the percentage of animals in each phenotypic class. Red box in ( $\mathrm{C}$ and $\mathrm{F}$ ) indicates the region between 0 and 1.5-fold change, which we consider to indicate no change in expression. All animals are shown in a lateral view with the oral side to the left. 
did not detect any ectodermal NvashA expression in 72 hpf animals. Animals treated with DAPT from 48 to 72 hpf showed a strong increase in NvashA in the forming pharynx and mesenteries (Figure 5E, arrow), and the endoderm has an increase in NvashA expression levels. As before, we could easily group phenotypic classes for NvashA expression: in control animals, $80 \%$ of the animals showed wild-type expression levels and only approximately 7\% showed the strong NvashA expression phenotype (Figure 5D). However, in the DAPT-treated animals $86 \%$ of the animals displayed the strong NvashA expression phenotype (Figure 5E). DAPT-treated animals also had an approximate three-fold increase in NvashA expression levels by qPCR (Figure 5F). These data demonstrate that DAPT treatment promotes an increase in NvashA at later stages, and that similar mechanisms regulate both embryonic and larval differentiation. Moreover, these results argue that the dynamic expression patterns observed for Nvnotch and Nvdelta (ectoderm in early embryo and moving into the endoderm in larval stages [26]) supports the hypothesis that Nvnotch regulates cellular differentiation in multiple tissues throughout development in Nematostella.

\section{Nvnotch broadly inhibits expression of genes associated with neuronal differentiation}

Lastly, we wondered if Notch activity might influence expression levels of other differentiation genes unrelated to NvashA. We used previously described differentiation genes, Nvgcm, Nvsoxb2, Nvsox2, Nvmef2.iv, and Nvminicol4 [31,38-40], as well as two recently identified genes, $N v$ coup 1 and Nvath-like1 (Figure 6), that, like NvashA, are all expressed in a "salt and pepper" pattern. It should be noted that all of these genes are associated with neuronal differentiation, though only Nvmef2.iv and Nvminicol4 have been definitively linked to neural development. They regulate formation of the cnidocyte neural cell type $[39,40]$. As we observed for NvashA, inhibiting Notch activity by treating with DAPT (Figure 6, blue bars) or injecting the Nvnotch MO (Additional file 4, green bars) increased expression levels for nearly all the "salt and pepper" genes assayed. The only genes assayed that showed no significant increase in expression levels following treatment with DAPT were Nvmef2.iv and Nvminicol4, though Nvminicol4 was upregulated following Nvnotch MO injection (Additional file 4). We also included Nvsox1, Nvsox3, Nvsoxe1, and Nvets1a because they are expressed in distinct broad domains rather than in a "salt and pepper" pattern, which suggests that they are involved in patterning regional domains rather than differentiation. Expression levels of the "broadly expressed" genes did not change following DAPT treatment or injection of the Nvnotch MO. Overactivation of Notch signaling by injecting Nvnicd:venus suppressed expression of all of the "salt

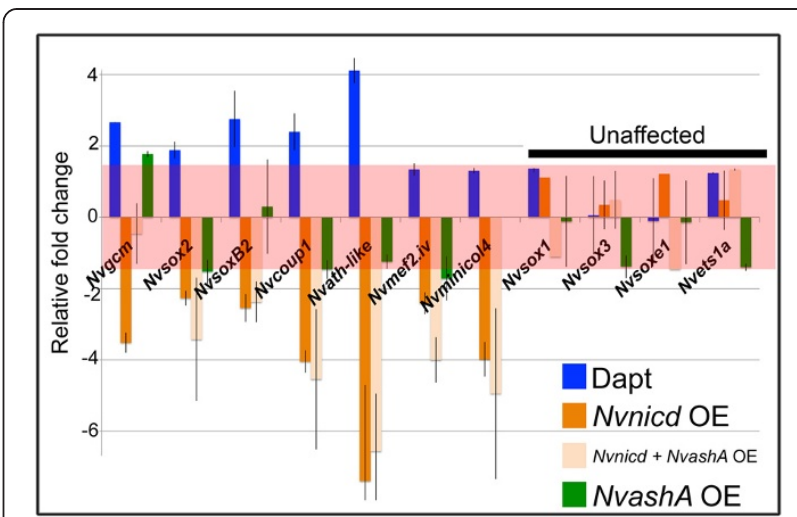

Figure 6 Nvnotch regulates "salt and pepper" differentiation genes. Relative fold change of "salt and pepper" genes in animals following treatment with DAPT (blue bars), injection with Nvnicd (dark orange bars), injection with Nvnicd and NvashA (light orange bars), or NvashA alone (green bars). Red rectangle denotes relative fold change -1.5 to 1.5 , which indicates no change in relative expression. "salt and pepper" differentiation genes are suppressed by Nvnotch activity while genes with broad expression domains are unaffected by any of our treatments. OE, overexpressing.

and pepper" genes (Figure 6, dark orange bars), including Nvmef2.iv and Nvminicol4. Again, the broadly expressed genes were unaffected by Nvnicd injection.

To confirm these genes are independent of NvashA, we attempted to rescue the loss of "salt and pepper" gene expression resulting from overactivation of Notch signaling by co-injecting the Nvnicd:venus and the NvashA: venus constructs (Figure 6, light orange bars). Only Nvgcm was rescued by expression of NvashA. This suggests that, with the exception of $N v g \mathrm{~cm}$, the "salt and pepper" genes are not targets of NvashA. Therefore, we suggest Notch activity broadly regulates expression of genes associated with neural differentiation in the Nematostella embryo.

\section{The non-canonical Notch signaling pathway inhibits NvashA expression}

Suppression of NvashA by activated Notch signaling can occur through the canonical (suH and hes genedependent), the non-canonical (suH and hes geneindependent), or through both pathways. We tested the putative contributions of the canonical and non-canonical pathways in Nematostella. First, we tested if Nvnotch regulated the expression of Nvhes genes. Four Nvhes genes, Nvhes1, 2, 3, Nvhl1, are expressed in Nematostella embryos and could potentially be regulating NvashA [26]. However, only Nvhes 2 and Nvhes3 expression is detected by mRNA in situ hybridization in the early gastrula when the earliest onset of differentiation of NvashA positive cells is occurring [26]. We compared changes in expression for each of these genes using qPCR following treatment with DAPT (Figure 7, blue bars), injection of the Nvnotch MO (Figure 7 , orange bars), and following injection of the 
Nvnicd:venus mRNA (Figure 7, purple bars). Treatment with DAPT resulted in an approximate two-fold reduction in Nvhes1 and Nvhl1 levels. The Nvhes2 and Nvhes3 genes both showed a greater than eight-fold reduction in expression following DAPT treatment (Figure 7A, blue bars). However, Nvnotch MO injected animals showed no change in Nvhes 1 or Nvhl1 expression, and a relatively minor decrease in Nvhes2 and Nvhes 3 levels (Figure 7A, orange bars). We failed to detect any reduction of Nvhes 2 or Nvhes3 in Nvnotch morphants by mRNA in situ hybridization (Figure 7B-E). Because the NvashA phenotype resulting from injection of the Nvnotch $\mathrm{MO}$ is as severe as treatment with DAPT (Figure 1), and there is little wild-type Nvnotch transcript in the Nvnotch morphant animals (Additional file 2A), we believe the Notch MO to be highly efficient. However, we were concerned that low levels of Nvnotch activity may be sufficient to promote Nvhes gene expression in the embryo. To address this we overactivated Notch signaling by injecting the Nvnicd:venus and Nvdelta:venus constructs, which should increase Nvhes expression if the canonical pathway was intact. We observed no significant change for Nvhes1-3 and only a minor increase in Nvhl1 expression following injection of Nvnicd:venus (Figure 7E, purple bars). Similarly, injection of the Nvdelta:venus mRNA failed to induce expression of any of the Nvhes genes. Thus, our data suggest that, although DAPT treatment reduces the expression levels of Nvhes1-3 or Nvhl1 in Nematostella embryos, the observed downregulation is Nvnotch-independent.

Even though Nvnotch does not regulate Nvhes genes we still wanted to test if NvsuH regulated Nvhes genes, and if Nvhes genes were sufficient to suppress NvashA expression. Nvhes 2 and Nvhes 3 are the only two Nvhes genes that have expression that initiates in the early embryo when the first cellular differentiation is observed in Nematostella. We overexpressed Nvhes 2 and Nvhes3 by injecting venus:Nvhes 2 and venus:Nvhes3 mRNAs. Nvhes2 or Nvhes3 overexpression did not result in any changes in the levels of NvashA as detected by mRNA in situ hybridization (Figure 8A-F) or qPCR (Figure 8G). We tested if $N v s u H$ regulated Nvhes genes or NvashA by injecting both an $\mathrm{NvsuH} \mathrm{MO}$ and a dominant negative $\mathrm{NvsuH}$ [26]. Neither of these manipulations resulted in detectable changes of Nvhes or NvashA expression by qPCR (Figure $8 \mathrm{H}$ ). These data suggest that the canonical Notch pathway does not regulate NvashA-dependent neural development in the early Nematostella embryo.

To determine if canonical Notch signaling could regulate the NvashA-independent "salt and pepper" expressed genes associated with cellular differentiation, we tested whether overexpressing Nvhes 2 or Nvhes 3 via injection of the venus:Nvhes 2 or venus:Nvhes 3 mRNA could suppress expression of the "salt and pepper" genes. We saw no change in the expression levels by qPCR for any of the "salt and pepper" genes assayed here (Additional file 4, light and dark blue bars). Thus, it appears that non-canonical Notch signaling broadly suppresses expression of genes that promote neural differentiation in Nematostella embryos.

\section{Discussion}

\section{Model of Notch signaling in Nematostella}

Our data show that NvNotch is activated by NvDelta to regulate cellular differentiation in Nematostella, but based on our observations here it is likely that Notch activity in

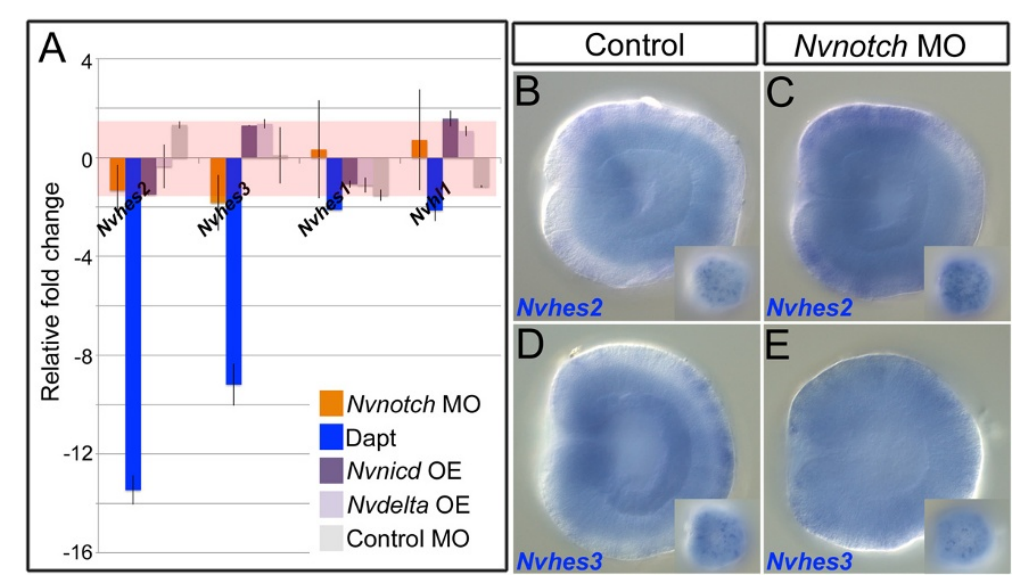

Figure 7 Nvnotch does not regulate Nvhes expression in the Nematostella embryo. (A) Average relative fold change of Nvhes gene expression in animals injected with Nvnotch morpholino (MO; orange bars), treated with DAPT (blue bars), injected with Nvnicd:venus (dark purple bars), injected with Nvdelta:venus (light purple bars), or a control MO (grey bars. Red rectangle covers the region where the relative fold change ratio is equal to -1.5 to 1.5 and corresponds to no change in relative expression level. (B-E) Lateral views of late stage gastrula expressing Nvhes2 (B-C) or Nvhes3 (D-E). Oral is to the left. Deep focal plane is shown and superficial focal plane is shown in inset. We observed no discernable difference in Nvhes2 or Nvhes3 expression by in situ analysis between wild-type and Nvnotch MO injected animals. We scored $\mathrm{N}>80$ embryos for each treatment. $\mathrm{OE}$, overexpressing. 


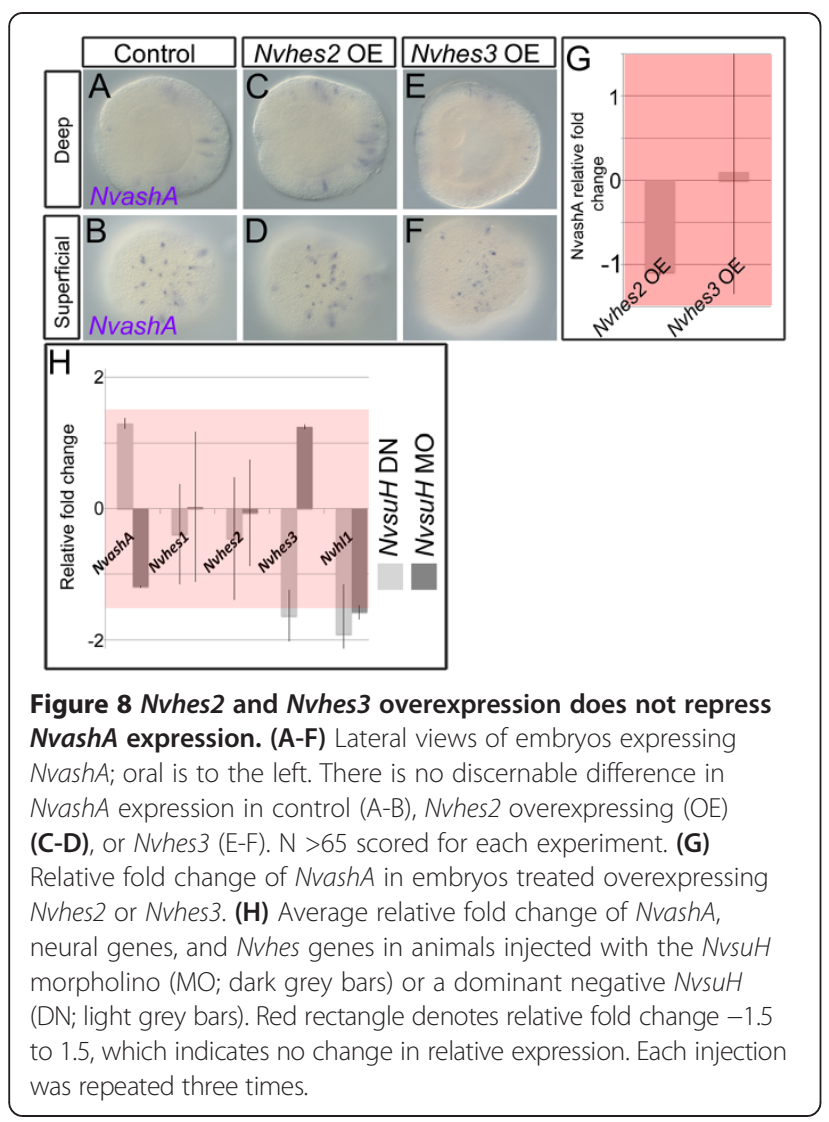

Nematostella regulates the competence of cells to respond to a variety of instructive differentiation cues. Elevated levels of Notch activity suppress differentiated cell markers, while decreased levels of Notch activity increase expression of differentiated cell markers (Figures 1 and 8; Additional file 1). However, inhibition of Notch signaling is not sufficient to induce a total transformation of cells into differentiated cells. This suggests that Notch either acts at defined time points in the differentiation process or that Notch-independent instructive cues act to induce particular differentiated cell types. Our model predicts that the relative level of Notch activity and the amount of inductive signal coordinate to determine if differentiation will occur. Consistent with this prediction, extending treatment with DAPT to 3 days results in animals that have a more pronounced expansion of differentiated cell markers than our early shorter treatment [26]. One interpretation is that extended inhibition of Notch activity provides more opportunity for undifferentiated cells to encounter and respond to external inductive cues. Additionally, quantification of the number of cells expressing any one "salt and pepper" gene is not often reproducible from animal to animal (Figure 1H; unpublished observations) [30], suggesting that the mechanism governing the number of cells of a distinct cell type is somewhat stochastic. Taken together these observations argue that, in any given animal at a given time, there are variable numbers of cells competent to respond to distinct differentiation cues. Our data supports the hypothesis that the competence is in part regulated by Nvnotch activity.

Notch appears to function broadly to inhibit neural differentiation. We tested a number of genes that have been previously reported to be associated with differentiation during Nematostella development (Figure 6; Additional file 3). We found that inhibiting Nvnotch by injecting the Nvnotch MO or by treating with DAPT resulted in upregulation of the differentiated markers. Conversely, overactivation of Notch by overexpressing the Nvnicd:venus mRNA suppressed expression of the differentiation markers. The markers that we used (NvashA, Nvsox2, Nvgcm, Nvsoxb2, Nvath-like, Nvmef2.iv, Nvminicol4, Nvcoup1-like) are all predicted to be associated with neurogenesis and/or cnidocyte development in Nematostella, although (with the exception of NvashA, Nvmef2.iv, and Nvminicol4) none of them are confirmed regulators of neural development. Thus, we cannot conclude at this point if Notch broadly regulates expression of all differentiated cell types or specifically regulates neural development in Nematostella. Even if the differentiation genes we chose are specific to neural development we argue that they are independent of NvashA-dependent neural development. We show that, other than $N v \mathrm{gcm}$, none of the differentiation genes assayed here can be rescued when Nvash $A$ is overexpressed in animals with increased Notch activity (Figure 5). Also, we have not observed any coexpression of $N v s o x b 2$ or $N v s o x 2$ with NvashA neural targets, and both $N v \operatorname{sox} B 2$ and $N v \operatorname{sox} 2$ are expressed in what appears to be many more cells than NvashA [38] (unpublished observation). Thus, we are confident that Notch activity broadly inhibits expression of genes associated with neural differentiation, but cannot determine what other cell types might be regulated by Notch activity.

We also propose that Notch regulation of differentiation is a reiterative process during Nematostella development. Differentiation begins during the early gastrula stage of Nematostella development, but continues throughout embryonic and larval stages. The expression patterns of Nvash $A$ and other known developmental genes are known to be dynamic throughout these stages $[30,31,36,38]$. Expression of Notch signaling components appears to be enriched in tissues likely to be undergoing cellular differentiation during development. For example, the embryonic expression of Nvnotch and Nvdelta initiate in the ectoderm, and are maintained there until late planula stages (Figure 2) [26]. In early planula stages the endoderm begins to show expression of differentiated cell markers $[31,36]$. Endodermal expression of Nvnotch and Nvdelta are coincident with endodermal differentiation. Nvnotch and Nvdelta are expressed in the forming and growing tentacle buds [26] (unpublished observation), and 
expression in juvenile and adult polyps is maintained in the endodermal portion of the eight mesenteries [26], where constant differentiation of nematosomes is known to occur [41]. We also found that treating with DAPT for distinct time windows throughout larval development resulted in the increased NvashA expression (Figure 5). This suggests that the same or a similar mechanism controls Nvash $A$ expression at later time points and in different tissues (endoderm versus ectoderm) than during embryonic development. We would like to extend this temporal analysis to gene-specific knockdowns of Nvnotch. However, we focused this initial study on the early embryonic roles of Nvnotch because conditional knockdown of Nvnotch function specifically at later time points is still difficult in Nematostella. As the technology of conditional alleles to disrupt gene function specifically at distinct life stages in Nematostella advances, and as identification of genes that serve as markers for cells differentiated within distinct temporal windows are found, our model can be tested further. We predict that disrupting Notch activity in distinct temporal windows should disrupt only the cell types that are normally born within that time frame.

\section{Notch signaling pathway may have emerged to regulate metazoan cellular differentiation}

The emergence of multicellular animals with specialized cell types had to require a mechanism to regulate whether cells differentiate or remain pluripotent. Notch has been shown to have a highly conserved role as a regulator of differentiation in nearly all bilaterian tissues. However, prior to this study it was unclear how Notch functioned in nonbilaterian animals, and thus there was little inference about ancestral Notch function. We show that non-canonical Notch signaling in the cnidarian sea anemone, Nematostella vectensis, broadly inhibits cellular differentiation during development. This provides a clear example of Notch regulating differentiation outside of Bilateria. Given how highly conserved the role for Notch as a regulator of differentiation appears, and the fact that core Notch components evolved specifically in metazoans, it is likely that Notch regulates differentiation in all metazoans. To fully support this hypothesis we need to reconstruct the function of Notch signaling in the common ancestor of all metazoans by characterizing the role of Notch in animals representing the earliest diverged metazoan lineage. The sister lineage to the rest of animals is still being debated, but the current consensus is that it is either Ctenophora or Porifera. Disruption of gene function in either of these groups has proven difficult, but we can infer putative function based on expression patterns. Expression of notch and delta homologs in the poriferan A. queenslandica initiates expression in a spatiotemporal pattern consistent with regulators of cellular differentation [25]. The amqdelta homologs appear to be expressed in differentiating and differentiated cell types consistent with the idea that they activate Notch to suppress differentiation in the surrounding cells, while having low Notch activity themselves [25]. The expression patterns of Notch signaling homologs in ctenophores are not known, and definitive homologs for delta have not been found. Thus, we cannot predict putative functions for Notch signaling in that lineage.

\section{Evolution of canonical Notch signaling}

Our results suggest that canonical Notch signaling is not present in the cnidarian lineage and that the canonical pathway evolved in the stem of the bilaterian lineage. In Nematostella, gene-specific knockdown of Nvnotch, NvsuH, or overactivation of Nvnicd did not significantly affect expression levels of Nvhes genes, which are an important target of the canonical Notch signaling pathway. Overactivation of Notch signaling by overexpressing either Nvnicd or Nvdelta was sufficient to suppress expression of differentiated cell markers, but both failed to upregulate any of the Nvhes genes monitored (Figure 5). Furthermore, overexpression of Nvhes 2 or Nvhes 3 failed to suppress NvashA or other genes associated with cellular differentiation (Figure 5; Additional file 4). In addition, the expression of Nvhes homologs throughout Nematostella development are inconsistent with the notion that they are targets of Nvnotch signaling. Most Nvhes genes show minimal overlap with Nvnotch expression outside of the embryonic ectoderm [26]. Three exceptions to this are Nvhes 3 and Nvhl1, which overlap with Nvnotch expression in the oral ectoderm and aboral ectoderm during planula stages [26], and Nvhes1, which overlaps with the Nvnotch expression in the planula endoderm. However, Nvhes1 expression appears ubiquitous in the planula stages, whereas Nvnotch expression becomes limited to the endoderm, suggesting that the Nvhes1 expression is regulated by factors other than Nvnotch. The reported expression of $\mathrm{NvsuH}$ is also inconsistent with the idea that canonical Notch signaling regulates differentiation. NvsuH is not expressed in the differentiating ectoderm at the onset of cellular differentiation in the early gastrula when expression of NvashA and the "salt and pepper" genes is initiated [26]. However, $N v s u H$ is expressed ubiquitously later in the planula larval stages.

A closer examination of the phylogenetic distribution of canonical Notch signaling components in the three published cnidarian genomes also supports the lack of an intact canonical Notch pathway in cnidarians $[4,23,24]$. Previous analysis suggested that the cnidarian-bilaterian common ancestor was the first animal with a compliment of genes that participate in canonical Notch signaling [19]. However, the cnidarian homologs of the transcriptional co-activator mastermind that is recruited to activate hes expression are only weakly conserved at best with bilaterian homologs 
$[1,19]$. Moreover, SuH also interacts with the SMRT corepressor to suppress expression of hes homologs when Notch signaling is not active. smrt homologs have not been identified in any of the currently published cnidarian genomes $[4,19,23,24]$.

It should be noted that most of the Nvhes genes are severely downregulated following DAPT treatment (Figure 5) [26]. However, our data argue that the DAPT-induced Nvhes phenotypes occur independently of Nvnotch. The current draft of the Nematostella genome describes only a single Nvnotch gene. However, there are additional single pass transmembrane proteins that, like Nvnotch, have EFG repeats in their extracellular domain (unpublished observation) [24]. The intracellular domains of these proteins lack the typical intracellular domains linking Notch signaling to hes gene regulation $[19,26]$, but because the $\gamma$-secretase complex is believed to cleave most single pass transmembrane signaling proteins, it is reasonable to hypothesize that DAPT is affecting one or more of these "Notch-like" proteins, and that they may regulate hes expression. Given that activation of hes expression is a hallmark of canonical Notch signaling, we speculate that some aspect of hes biology underlies the emergence of the canonical pathway. One explanation could be based on the fact that hes genes function as oscillators that promote cell proliferation [13,42]. Interestingly, we observe Nvhes 2 expression in proliferating cells (unpublished observation). Because high Notch activity often suppresses differentiation, perhaps incorporating regulation of proliferation downstream of Notch activity provided a mechanism to both suppress differentiation and promote proliferation. This is consistent with the observation that canonical Notch activity in the development of many bilaterian tissues is often associated with maintaining tissue-specific stem cells [8].

To verify that canonical Notch signaling is not intact in the cnidarian-bilaterian ancestor gene specific functional studies need to be conducted in other cnidarian species. Additional analyses need to be done in Nematostella once tools emerge to investigate roles for Notch signaling specifically during post-embryonic development. Currently, attempting to interpret late-stage phenotypes in morphant animals is complicated because it is unclear how early disruption of Nvnotch influences later development. Temporal-specific treatments with DAPT would not be informative because we showed that the responses of Nvhes genes to DAPT in the embryo are Nvnotchindependent phenotypes.

\section{Conclusions}

Based on our functional analysis in the cnidarian Nematostella vectensis and previous pharmacological experiments in other cnidarian species, we propose that the Notch signaling pathway regulated cellular differentiation in the cnidarian-bilaterian ancestor. This argues that the role of Notch as a regulator of cellular differentiation evolved prior to the last common ancestor of bilaterian animals. Functional studies are required in other nonbilaterian lineages to reconstruct the role of Notch signaling at more basal nodes in the metazoan phylogeny. Because all components of canonical Notch signaling likely did not evolve until the cnidarian-bilaterian common ancestor, a full complement of canonical signaling components only exists in the bilaterians, and because canonical Notch signaling is not required for Nvnotch to regulate embryonic neural differentiation in Nematostella, we speculate that non-canonical Notch signaling is the ancestral notch mechanism and that the canonical pathway likely evolved specifically in the bilaterian lineage.

\section{Additional files}

Additional file 1: DAPT treatment upregulates NvashA. (A-D) Shown are lateral views of embryos expressing NvashA. Oral is to the left. DAPT-treated animals have higher levels of NvashA expression. Phenotypic classes we scored as being wild-type, strong, weak, or no NvashA expression. Key is shown in image and bars at the base of each image represent the percentage of animals in each phenotypic class.

Additional file 2: Control experiments. (A) Splice blocking efficiency for each splice MO used in this study is shown. (B-D) Injection of mRNAs encoding for the Nvnicd:venus (B), venus:Nvhes2 (C), and venus:Nvhes3 (D) resulted in translated protein and can be detected in the nuclei of the developing embryo.

Additional file 3: Relative fold change of NvashA neuronal targets in Nvdelta OE animals. Relative fold change of NvashA neural target genes in animals overexpressing the Nvdelta:venus mRNA (light grey bars) or overexpressing the Nvdelta:venus mRNA and treated with DAPT (dark grey bars). Red box indicates region where fold change ratio is between -1.5 and 1.5 indicating no change in expression.

Additional file 4: Relative fold change of "salt and pepper" genes in Nvnotch morphant and Nvhes overexpressing animals. Relative

fold change of "salt and pepper" and broad domain expressed controls are shown for animals injected with the Nvnotch MO (green bars), venus: Nvhes2 (light blue bars), or venus:Nvhes3 (dark blue bars). Each injection was repeated at least three times. Red box indicates region where fold change ratio is between -1.5 and 1.5 indicating no change in expression.

\section{Abbreviations}

hpf: Hours post-fertilization; MO: morpholino; (q)PCR: (quantitative) polymerase chain reaction.

\section{Competing interests}

The authors declare that they have no competing interests.

\section{Authors' contributions}

MJL conceived and carried out the generation of constructs, collection of data, and data analysis. MJL and MQM carried out animal injections. MJL and MQM wrote the manuscript. Both authors read and approved the final manuscript.

\section{Acknowledgements}

We wish to acknowledge Dr Leslie Babonis, Dr Timothy Dubuc, and Dr Joseph Ryan for their critical reading of this manuscript. This work was funded by grant number R21RR034343 from the National Institutes of Health. 


\section{Author details}

'Department of Biological Sciences, Lehigh University, Bethlehem, PA 18015, USA. ${ }^{2}$ Whitney Laboratory for Marine Bioscience, University of Florida, St Augustine, FL 32080, USA.

Received: 27 May 2014 Accepted: 12 August 2014 Published: 19 September 2014

\section{References}

1. Ryan JF, Pang K, Schnitzler CE, Nguyen AD, Moreland RT, Simmons DK, Koch BJ, Francis WR, Havlak P, Comparative Sequencing Program NISC, Smith SA, Putnam NH, Haddock SHD, Dunn CW, Wolfsberg TG, Mullikin JC, Martindale MQ, Baxevanis AD: The genome of the ctenophore mnemiopsis leidyi and its implications for cell type evolution. Science 2013, 342: doi:1126/science. 124592

2. Dunn CW, Hejnol A, Matus DQ, Pang K, Browne WE, Smith SA, Seaver E, Rouse GW, Obst M, Edgecombe GD, Sørensen MV, Haddock SHD, SchmidtRhaesa A, Okusu A, Kristensen RM, Wheeler WC, Martindale MQ, Giribet G: Broad phylogenomic sampling improves resolution of the animal tree of life. Nature 2008, 452:745-749.

3. Srivastava M, Begovic E, Chapman J, Putnam NH, Hellsten U, Kawashima T, Kuo A, Mitros T, Salamov A, Carpenter ML, Signorovitch AY, Moreno MA, Kamm K, Grimwood J, Schmutz J, Shapiro H, Grigoriev IV, Buss LW, Schierwater B, Dellaporta SL, Rokhsar DS: The Trichoplax genome and the nature of placozoans. Nature 2008, 454:955-960.

4. Srivastava M, Simakov O, Chapman J, Fahey B, Gauthier MEA, Mitros T, Richards GS, Conaco C, Dacre M, Hellsten U, Larroux C, Putnam NH, Stanke M, Adamska M, Darling A, Degnan SM, Oakley TH, Plachetzki DC, Zhai Y, Adamski M, Calcino A, Cummins SF, Goodstein DM, Harris C, Jackson DJ, Leys SP, Shu S, Woodcroft BJ, Vervoort M, Kosik KS, et al: The Amphimedon queenslandica genome and the evolution of animal complexity. Nature 2010, 466:720-726

5. Liu J, Sato C, Cerletti M, Wagers A: Notch Signaling in the Regulation of Stem Cell Self-Renewal and Differentiation. Curr Top Dev Biol. 2010, 92:367-409.

6. Shawber C, Nofziger D, Hsieh JJD, Lindsell C, Bogler O, Hayward D, Weinmaster G: Notch signaling inhibits muscle cell differentiation through a CBF1-independent pathway. Development 1996, 122:3765-3773.

7. Ye Y, Lukinova N, Fortini ME: Neurogenic phenotypes and altered Notch processing in Drosophila presenilin mutants. Nature 1999, 398:525-529.

8. Koch U, Lehal R, Radtke F: Stem cells living with a Notch. Development 2013, 140:689-704.

9. Bray SJ: Notch signalling: a simple pathway becomes complex. Nat Rev Mol Cell Biol 2006, 7:678-689.

10. Simpson P: Notch and the choice of cell fate in Drosophila neuroepithelium. Trends Genet 1990, 6:343-345.

11. Skeath JB: At the nexus between pattern formation and cell-type specification: the generation of individual neuroblast fates in the Drosophila embryonic central nervous system. Bioessays 1999, 21:922-931.

12. Artavanis-Tsakonas S, Simpson P: Choosing a cell fate: a view from the Notch locus. Trends Genet 1991, 7:403-408.

13. Shimojo H, Ohtsuka T, Kageyama R: Oscillations in notch signaling regulate maintenance of neural progenitors. Neuron 2008, 58:52-64.

14. Cubas P, De Celis JF, Campuzano S: Proneural clusters of achaete-scute expression and the generation of sensory organs in the Drosophila imaginal wing disc. Genes \& Dev 1991, 5:996-1008.

15. Bertrand N, Castro DS, Guillemot F: Proneural genes and the specification of neural cell types. Nat Rev Neurosci 2002, 3:1-14.

16. Le Gall M, De Mattei C, Giniger E: Molecular separation of two signaling pathways for the receptor, Notch. Dev Biol 2008, 313:556-567.

17. Sanalkumar R, Dhanesh SB, James J: Non-canonical activation of Notch signaling/target genes in vertebrates. Cell Mol Life Sci 2010, 67:2957-2968.

18. Lecourtois M, Schweisguth F: The neurogenic suppressor of hairless DNAbinding protein mediates the transcriptional activation of the enhancer of split complex genes triggered by Notch signaling. Genes Dev 1995 , 9:2598-2608.

19. Gazave E, Lapébie P, Richards GS, Brunet F, Ereskovsky AV, Degnan BM, Borchiellini C, Vervoort M, Renard E: Origin and evolution of the Notch signalling pathway: an overview from eukaryotic genomes. BMC Evol Biol 2009, 9:249.
20. Heitzler P: Biodiversity and Noncanonical Notch Signaling. Curr Top Dev Biol 2010, 92:457-481.

21. Galliot B, Quiquand M, Ghila L, de Rosa R, Miljkovic-Licina M, Chera S Origins of neurogenesis, a cnidarian view. Dev Biol 2009, 332:2-24.

22. D'Souza B, Meloty-Kapella L, Weinmaster G: Canonical and Non-Canonical Notch Ligands. Curr Top Dev Biol 2010, 92:73-129.

23. Chapman JA, Kirkness EF, Simakov O, Hampson SE, Mitros T, Weinmaier T, Rattei T, Balasubramanian PG, Borman J, Busam D, Disbennett K, Pfannkoch C, Sumin N, Sutton GG, Viswanathan LD, Walenz B, Goodstein DM, Hellsten U, Kawashima T, Prochnik SE, Putnam NH, Shu S, Blumberg B, Dana CE, Gee L, Kibler DF, Law L, Lindgens D, Martinez DE, Peng J, et al: The dynamic genome of Hydra. Nature 2010, 464:592-596.

24. Putnam NH, Srivastava M, Hellsten U, Dirks B, Chapman J, Salamov A, Terry A, Shapiro H, Lindquist E, Kapitonov W, Jurka J, Genikhovich G, Grigoriev IV, Lucas SM, Steele RE, Finnerty JR, Technau U, Martindale $M Q$, Rokhsar DS: Sea anemone genome reveals ancestral eumetazoan gene repertoire and genomic organization. Science 2007, 317:86-94.

25. Richards GS, Degnan BM: The expression of Delta ligands in the sponge Amphimedon queenslandica suggests an ancient role for Notch signaling in metazoan development. Evodevo 2012, 3:1.

26. Marlow H, Roettinger $E$, Boekhout M, Martindale MQ: Functional roles of Notch signaling in the cnidarian Nematostella vectensis. Dev Biol 2012, 362:1-14.

27. Münder S, Tischer S, Grundhuber M, Büchels N, Bruckmeier N, Eckert S, Seefeldt CA, Prexl A, Käsbauer T, Böttger A: Notch-signaling is required for head regeneration and tentacle patterning in Hydra. Dev Biol 2013, 383:146-157.

28. Käsbauer T, Towb P, Alexandrova O, David CN, Dall'Armi E, Staudigl A, Stiening $B$, Böttger $A$ : The Notch signaling pathway in the cnidarian Hydra. Dev Biol 2007, 303:376-390.

29. Dovey HF, John V, Anderson JP, Chen LZ, de Saint AP, Fang LY, Freedman SB, Folmer B, Goldbach E, Holsztynska EJ, Hu KL, JohnsonWood KL, Kennedy SL, Kholodenko D, Knops JE, Latimer LH, Lee M, Liao Z, Lieberburg IM, Motter RN, Mutter LC, Nietz J, Quinn KP, Sacchi KL, Seubert PA, Shopp GM, Thorsett ED, Tung JS, Wu J, Yang S, et al: Functional gamma-secretase inhibitors reduce beta-amyloid peptide levels in brain. J Neurochem 2001, 76:173-181.

30. Layden MJ, Boekhout M, Martindale MQ: Nematostella vectensis achaete-scute homolog NvashA regulates embryonic ectodermal neurogenesis and represents an ancient component of the metazoan neural specification pathway. Development 2012, 139:1013-1022.

31. Marlow HQ, Srivastava M, Matus DQ, Rokhsar D, Martindale MQ: Anatomy and development of the nervous system of Nematostella vectensis, an anthozoan cnidarian. Devel Neurobio 2009, 69:235-254.

32. Layden MJ, Meyer NP, Pang K, Seaver EC, Martindale MQ: Expression and phylogenetic analysis of the zic gene family in the evolution and development of metazoans. Evodevo 2010, 1:12

33. Wolenski FS, Layden MJ, Martindale MQ, Gilmore TD, Finnerty JR: Characterizing the spatiotemporal expression of RNAs and proteins in the starlet sea anemone, Nematostella vectensis. Nat Protoc 2013, 8:900-915.

34. Layden MJ, Röttinger E, Wolenski FS, Gilmore TD, Martindale MQ: Microinjection of mRNA or morpholinos for reverse genetic analysis in the starlet sea anemone, Nematostella vectensis. Nat Protoc 2013, 8:924-934.

35. Sinigaglia $\mathrm{C}$, Busengdal $H$, Leclère $L$, Technau U, Rentzsch F: The bilaterian head patterning gene six $3 / 6$ controls aboral domain development in a cnidarian. Plos Biol 2013, 11:e1001488

36. Nakanishi N, Renfer $E$, Technau U, Rentzsch F: Nervous systems of the sea anemone Nematostella vectensis are generated by ectoderm and endoderm and shaped by distinct mechanisms. Development 2011, 139:347-357.

37. Kopan R, Nye JS, Weintraub H: The intracellular domain of mouse Notch: a constitutively activated repressor of myogenesis directed at the basic helix-loop-helix region of MyoD. Development 1994 $120: 2385-2396$

38. Magie CR, Pang K, Martindale MQ: Genomic inventory and expression of Sox and Fox genes in the cnidarian Nematostella vectensis. Dev Genes Evol 2005, 215:618-630.

39. Zenkert C, Takahashi T, Diesner MO, Ozbek S: Morphological and molecular analysis of the nematostella vectensis cnidom. PLOS One 2011, 6:e22725. 
40. Genikhovich G, Technau U: Complex functions of Mef2 splice variants in the differentiation of endoderm and of a neuronal cell type in a sea anemone. Development 2011, 138:4911-4919.

41. Williams RB: Studies on the nematosomes of Nematostella vectensis Stephenson (coelenterata: Actiniaria). J Nat Hist 1979, 13:60-80.

42. Kageyama R, Ohtsuka $T$, Kobayashi $T$ : The Hes gene family: repressors and oscillators that orchestrate embryogenesis. Development 2007, 134:1243-1251.

doi:10.1186/2041-9139-5-30

Cite this article as: Layden and Martindale: Non-canonical Notch signaling represents an ancestral mechanism to regulate neural differentiation. EvoDevo 2014 5:30.

\section{Submit your next manuscript to BioMed Central and take full advantage of:}

- Convenient online submission

- Thorough peer review

- No space constraints or color figure charges

- Immediate publication on acceptance

- Inclusion in PubMed, CAS, Scopus and Google Scholar

- Research which is freely available for redistribution 